\title{
Top-down Holographic Glueball Decay Rates
}

\author{
F. Brünner, D. Parganlija and A. Rebhan \\ Institut für Theoretische Physik, Technische Universität Wien, \\ Wiedner Hauptstrasse 8-10, A-1040 Vienna, Austria
}

\begin{abstract}
We present new results on the decay patterns of scalar and tensor glueballs in the top-down holographic WittenSakai-Sugimoto model. This model, which has only one free dimensionless parameter, gives semi-quantitative predictions for the vector meson spectrum, their decay widths, and also a gluon condensate in agreement with SVZ sum rules. The holographic predictions for scalar glueball decay rates are compared with experimental data for the widely discussed gluon candidates $f_{0}(1500)$ and $f_{0}(1710)$.
\end{abstract}

Keywords: Gauge-gravity duality, AdS/CFT correspondence, AdS/QCD, QCD, glueballs, gluonium, mesons PACS: $11.25 . \mathrm{Tq}, 13.25 . \mathrm{Jx}, 14.40 . \mathrm{Be}, 14.40 . \mathrm{Rt}$

\section{INTRODUCTION}

Glueballs, color-neutral bound states of gluons, are the only physical states available in pure Yang-Mills theory and are also expected to show up in the meson spectrum of quantum chromodynamics (QCD) [1,2,3]. The mass of the lowest glueball state with $J^{P C}=0^{++}$is predicted by lattice gauge theory $[4,5,6,7]$ to be in the range $1.5-1.8 \mathrm{GeV}$. However, as reviewed in Refs. $[8,9,10]$, the identification of glueballs in the meson spectrum has remained elusive and will be in the focus of the PANDA experiment at FAIR [11, 12]. Various scenarios have been proposed, from unobservably broad glueball resonances $[13,14]$ to narrow glueballs that are identified with a dominant component of one of the fairly narrow isoscalar mesons in the mass range of the lattice results, $f_{0}(1500)$ or $f_{0}(1710)[15,16,17,18,19,20,21]$.

QCD sum rules seem to require a broad scalar glueball $[13,21]$, whereas the usually quite reliable large- $N_{c}$ limit would indicate narrow states. The situation is similarly difficult in the case of tensor glueballs, which lattice simulations predict to occur in the range 2.2-2.6 GeV, but a clear identification of a corresponding $f_{2}$ meson is missing.

More information from first-principle approaches on the properties of glueballs would clearly be helpful. Lattice gauge theory should also be able to provide information on decay rates, but the extraction of real-time quantities is difficult, and glueballs are particularly difficult to investigate in the presence of dynamical quarks.

\section{HOLOGRAPHIC GLUEBALL SPECTRUM IN THE WITTEN MODEL}

A new approach to study glueballs and their properties has become available with the advent of gauge-gravity constructions where the supersymmetry and conformal symmetry of the underlying AdS/CFT correspondence is broken. The first and still most important example of a top-down holographic approach towards (large- $N_{c}$ ) QCD is the Witten model [22,23] which is based on $N_{c}$ D4 branes in type-IIA supergravity where one spatial dimension is compactified on a Kaluza-Klein circle with antiperiodic boundary conditions for the fermionic gauginos. This not only renders the gauginos massive, but through loop corrections also all adjoint scalar matter, so that in the limit where the Kaluza-Klein mass is sent to infinity one would arrive at pure-glue large- $N_{c}$ Yang-Mills theory. Unfortunately, this limit is not accessible without leaving the realm of supergravity and going over to a full gauge-string duality which is too difficult for being of practical use. However, the hope is that already the supergravity approximation allows one to study nonperturbative features of large- $N_{c}$ gauge theories qualitatively and that perhaps certain quantitative results are not too far from those of actual QCD, which is indeed frequently approximated quite well by large- $N_{c}$ results.

The supergravity background of the Witten model can be obtained by another dimensional reduction, namely a supersymmetry-preserving circle compactification of 11-dimensional supergravity with $x^{11} \simeq x^{11}+2 \pi R_{11}$, which turns the AdS/CFT correspondence available for M5 branes to a nonconformal gauge-gravity correspondence for D4 branes. After introducing the supersymmetry-breaking circle compactification $x^{4} \simeq x^{4}+2 \pi R_{4}, R_{4} \equiv M_{\mathrm{KK}}^{-1}$, the gravitational 
$\mathrm{M} / \mathrm{M}_{\mathrm{KK}}$

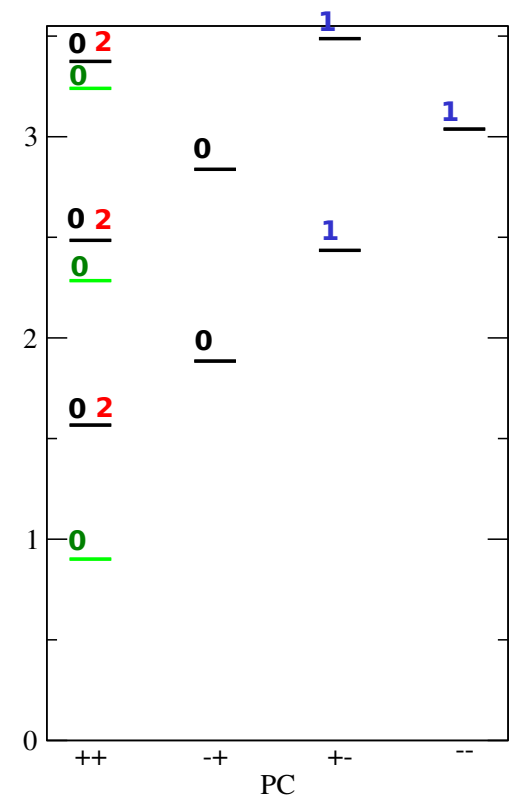

$\mathrm{M} / \sqrt{ } \sigma$

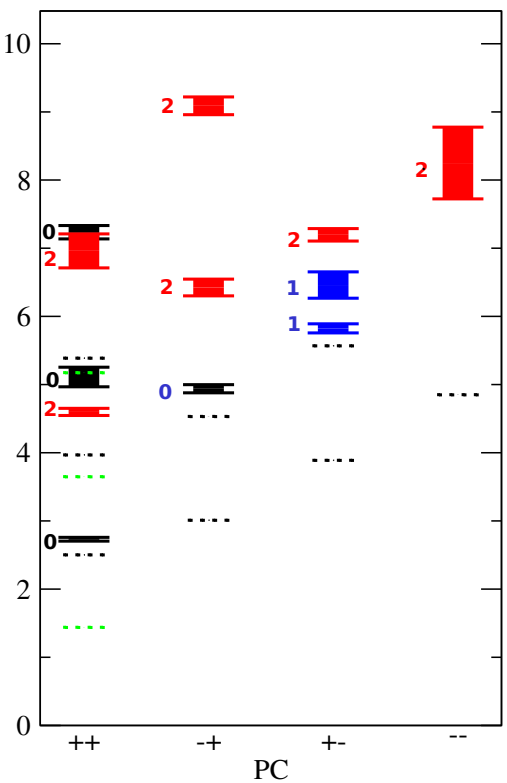

FIGURE 1. The holographic glueball spectrum of the Witten model (left panel, masses in units of $M_{\mathrm{KK}}$, "exotic" scalar mode in green) compared to the recent large- $N_{c}$ lattice results of Ref. [24] (right panel, masses in units of the square root of the string tension). Dotted lines give the holographic glueball masses divided by $\sqrt{\sigma}$ from (11) with $\lambda=16.63$.

background is given by a doubly Wick-rotated $\mathrm{AdS}_{7}$ black hole geometry with (11-dimensional) metric

$$
\begin{gathered}
d s^{2}=\frac{r^{2}}{L^{2}}\left(f(r) d x_{4}^{2}+\eta_{\mu v} d x^{\mu} d x^{v}+d x_{11}^{2}\right)+\frac{L^{2}}{r^{2}} \frac{d r^{2}}{f(r)}+\frac{L^{2}}{4} d \Omega_{4}^{2}, \\
f(r)=1-\frac{r_{\mathrm{KK}}^{6}}{r^{6}}, \quad r_{\mathrm{KK}}=\frac{L^{2}}{3} M_{\mathrm{KK}},
\end{gathered}
$$

where $L$ is the curvature radius of the asymptotic $\mathrm{AdS}_{7}$ space forming a product space with an $S^{4}$ of radius $L / 2$. With $x^{4}$ and $x^{11}$ compactified, the boundary theory at sufficiently low energies is 4-dimensional Yang-Mills theory with parameters

$$
g_{\mathrm{YM}}^{2}=\frac{g_{5}^{2}}{2 \pi R_{4}}=2 \pi g_{s} l_{s} M_{\mathrm{KK}}, \quad(L / 2)^{3} \equiv R_{\mathrm{D} 4}^{3}=\pi g_{s} N_{c} l_{s}^{3} .
$$

Here $g_{\mathrm{YM}}$ is the Yang-Mills coupling at the scale $M_{\mathrm{KK}}$, normalized such that the QCD coupling $\alpha_{s} \equiv g^{2} /(4 \pi)=$ $g_{\mathrm{YM}}^{2} /(2 \pi)$ (see Ref. [25] for a discussion of this point).

One of the earliest applications of gauge/gravity duality has been the calculation of the glueball spectrum from the supergravity background of the Witten model $[26,27,28,29,30]$. For this one needs to determine the spectrum of fluctuations of the metric and the 3-form field of the 11-dimensional supergravity theory. When classified according to their symmetries in the noncompact dimensions $x^{0}, \ldots, x^{3}$ of the boundary theory this yields towers of scalar, vector, and tensor glueballs. This has been worked out completely in Ref. [30], with results displayed in Fig. 1 and compared with recent lattice results for the glueball spectrum in large- $N_{c}$ gauge theory. Simpler (bottom-up) models [31, 32, 33] typically have fewer towers of glueballs, which include scalar glueballs dual to the dilaton, and tensor glueballs dual to the metric field.

In the Witten model, the dilaton mode of type-IIA supergravity arises as

$$
\delta \Phi=\frac{3 L^{2}}{4 r^{2}} \delta G_{11,11}
$$


A scalar mode is obtained by having fluctuations of the form

$$
\begin{aligned}
\delta G_{11,11} & =-3 \frac{r^{2}}{L^{2}} H_{D}(r) G_{D}(x), \\
\delta G_{\mu \nu} & =\frac{r^{2}}{L^{2}} H_{D}(r)\left[\eta_{\mu \nu}-\frac{\partial_{\mu} \partial_{v}}{\square}\right] G_{D}(x),
\end{aligned}
$$

which also involves the trace part of metric fluctuations. The mass of this mode turns out to be degenerate with tensor modes from transverse-traceless fluctuations of only $\delta G_{\mu \nu}$.

As noted first in Ref. [29], the Witten model has another scalar mode associated with fluctuations involving also $\delta G_{44}$, a metric fluctuation with components pertaining to the compactification dimension:

$$
\begin{aligned}
& \delta G_{44}=-\frac{r^{2}}{L^{2}} f H_{G}(r) G(x) \\
& \delta G_{\mu \nu}=\frac{r^{2}}{L^{2}} H_{G}(r)\left[\frac{1}{4} \eta_{\mu \nu}-\left(\frac{1}{4}+\frac{3 r_{\mathrm{KK}}^{6}}{5 r^{6}-2 r_{\mathrm{KK}}^{6}}\right) \frac{\partial_{\mu} \partial_{\nu}}{M_{E}^{2}}\right] G(x), \\
& \delta G_{11,11}=\frac{r^{2}}{L^{2}} \frac{1}{4} H_{G}(r) G(x), \\
& \delta G_{r r}=-\frac{L^{2}}{r^{2}} f^{-1} \frac{3 r_{\mathrm{KK}}^{6}}{5 r^{6}-2 r_{\mathrm{KK}}^{6}} H_{G}(r) G(x), \\
& \delta G_{r \mu}=\frac{90 r^{7} r_{\mathrm{KK}}^{6}}{M_{E}^{2} L^{2}\left(5 r^{6}-2 r_{\mathrm{KK}}^{6}\right)^{2}} H_{G}(r) \partial_{\mu} G(x) .
\end{aligned}
$$

Ref. [30] subsequently found that this "exotic polarization" [29] has the smallest mass of all the supergravity modes.

As displayed in Fig. 1, this does make the overall spectrum of the holographic glueballs appear to qualitatively agree with what is found in lattice gauge theory, but it leaves a certain abundance of scalar modes in the $J^{P C}=0^{++}$sector. Because the mass scale $M_{\mathrm{KK}}$ is not fixed in the Witten model, a more quantitative comparison with the lattice results is not possible. However, this changes when one extends the model to incorporate also quarks.

\section{INTERACTIONS WITH CHIRAL QUARKS AND MESONS IN THE SAKAI-SUGIMOTO MODEL}

In 2004, the Witten model was extended by Sakai and Sugimoto $[34,35]$ by a configuration of probe D8 and anti-D8 branes that fill all of the 10-dimensional space of the Witten model except for the circle along $x^{4}$. This introduces $N_{f} \ll N_{c}$ chiral quarks and antiquarks that are localized in $x^{4}$, but since the D8 and anti-D8 branes have nowhere to end in the subspace spanned by $x^{4}$ and the radial (holographic) direction, these branes have to connect in the bulk geometry, leading to a simple geometric realization of chiral symmetry breaking (see Fig. 2).

The low-energy theory of the (Witten-)Sakai-Sugimoto model involves a chiral Lagrangian for the NambuGoldstone bosons with a Skyrme term, which reflects the presence of a baryon vertex provided by additional D4 branes wrapping the $S^{4}$ with $N_{c}$ units of Ramond flux. It also provides the correct Wess-Zumino-Witten term required by the chiral anomaly of QCD. With a minimal set of parameters, it represents arguably the most intriguing model of holographic QCD. As reviewed in Ref. [25], several features of the low-energy spectrum of QCD are nicely reproduced.

Here we shall discuss in particular the predictions of the Witten-Sakai-Sugimoto model for the interactions of glueballs with mesons composed of chiral quarks, which were first studied in [36] and revisited by us in Ref. [37, 38].

Such interactions are determined by the action of the flavor D8 and anti-D8 branes,

$$
\begin{aligned}
S_{\mathrm{D} 8} & =-T_{\mathrm{D} 8} \operatorname{Tr} \int d^{9} x e^{-\Phi} \sqrt{-\operatorname{det}\left(\tilde{g}_{M N}+2 \pi \alpha^{\prime} F_{M N}\right)}+S_{\mathrm{CS}} \\
& =-\left(2 \pi \alpha^{\prime}\right)^{2} T_{\mathrm{D} 8} \operatorname{Tr} \int d^{9} x e^{-\Phi} \sqrt{-\tilde{g}}\left(\mathbf{1}+\frac{1}{4} \tilde{g}^{P R} \tilde{g}^{Q S} F_{P Q} F_{R S}+O\left(F^{4}\right)\right)+S_{\mathrm{CS}}
\end{aligned}
$$



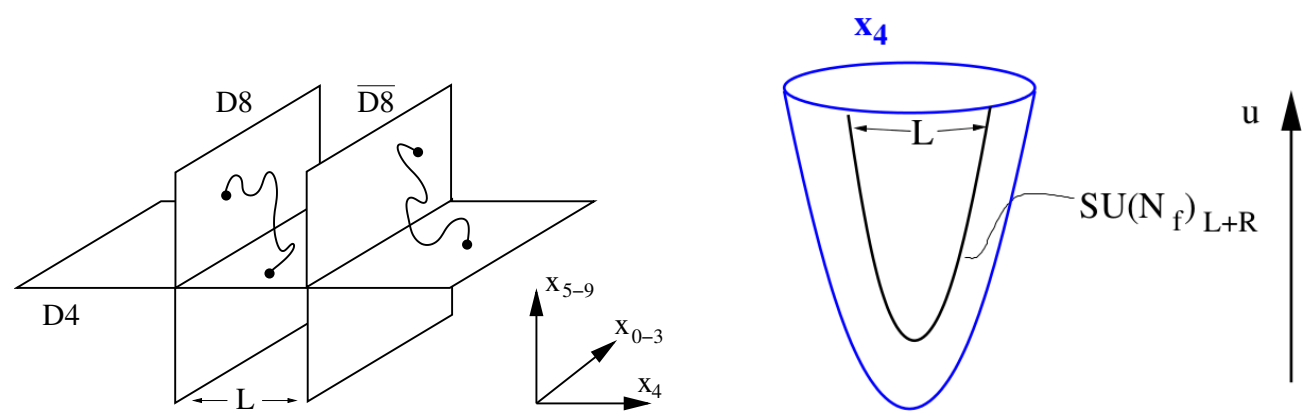

FIGURE 2. Left: Configuration of the stacks of $N_{c}$ D4 branes and $N_{f}$ D8 and $\overline{\mathrm{D} 8}$ branes in the 10 dimensions $x_{0}, \ldots, x_{9}$, with $x_{4}$ taken as periodic. Right: Geometry near the horizon of the stack of $N_{c}$ D4 branes, $N_{c} \gg N_{f}$, with $u$ the radial coordinate in the transverse space $x_{5 . . .9}$. [Figure courtesy of Andreas Schmitt]

where the leading-order terms are explicitly given by

$$
S_{\mathrm{D} 8}^{\left(F^{2}\right)}=-\kappa \operatorname{Tr} \int d^{4} x \int_{-\infty}^{\infty} d Z\left[\frac{1}{2} K^{-1 / 3} \eta^{\mu \rho} \eta^{\nu \sigma} F_{\mu \nu} F_{\rho \sigma}+M_{\mathrm{KK}}^{2} \eta^{\mu \nu} F_{\mu Z} F_{v Z}\right], \quad \kappa=\frac{\lambda N_{c}}{216 \pi^{3}},
$$

with $K \equiv 1+Z^{2}$, where $Z$ is a dimensionless holographic coordinate that runs from $-\infty$ to $\infty$ as the connected branes (antipodal in $x^{4}$ ) are followed from the boundary to the minimal value of the holographic radial coordinate $r$ and back along the oppositely charged brane to the boundary.

The global chiral symmetry $\mathrm{SU}\left(N_{f}\right) \times \mathrm{SU}\left(N_{f}\right)$ corresponds to a gauge symmetry with flavor gauge fields on the D8 branes. Its spontaneous breaking is reflected by a massless mode $A_{Z}=\phi_{0}(Z) \pi(x)$ containing the Nambu-Goldstone bosons; massive vector and axial vector mesons are described by the even and odd eigenmodes of $A_{\mu}^{(n)}=\psi_{n}(Z) v_{\mu}^{(n)}(x)$. The lowest eigenvalue gives the identification

$$
m_{\rho}^{2}=0.699314 \ldots M_{\mathrm{KK}}^{2} .
$$

The real-world value of $m_{\rho}$ can therefore be used to set the overall mass scale of the Witten-Sakai-Sugimoto model, leading to $M_{\mathrm{KK}}=949 \mathrm{MeV}$. The masses of higher vector and axial vector mesons as predicted by the eigenvalue equation for $\psi_{n}(Z)$ are in fact surprisingly close to experimental results. For example, the predicted ratios $m_{a_{1}} / m_{\rho}$ and $m_{\rho^{*}} / m_{\rho}$ deviate only by $4 \%$ and $10 \%$ from the measured values. This agreement may however be somewhat fortuitous, for recent lattice simulations [39] at large $N_{c}$, where the holographic model should actually become more reliable, lead to deviations by $21 \%$ and $16 \%$, respectively. Still, this can be viewed as a remarkable success given that the WittenSakai-Sugimoto model should show deviations from large- $N_{c}$ QCD at scales above $M_{\mathrm{KK}}$ and that already $v_{\mu}^{(2)}$ is above $M_{\mathrm{KK}}$.

Other predictions of the Witten-Sakai-Sugimoto model for $N_{c}=3 \mathrm{QCD}$ depend on the value of the 't Hooft coupling at the scale $M_{\mathrm{KK}}$ above which the model turns into 5-dimensional super-Yang-Mills theory. In the original papers $[34,35]$ the pion decay constant

$$
f_{\pi}^{2}=\frac{1}{54 \pi^{4}} \lambda N_{c} M_{\mathrm{KK}}^{2}
$$

was fixed to $f_{\pi} \approx 92.4 \mathrm{MeV}$, which together with $M_{\mathrm{KK}}=949 \mathrm{MeV}$ determines the constant $\kappa$ in (7) as

$$
\kappa \equiv \lambda N_{c} /\left(216 \pi^{3}\right)=7.45 \cdot 10^{-3}
$$

corresponding to $\lambda=16.63$.

As reviewed in Ref. [25], most other possibilities of fixing $\lambda$ point to somewhat smaller values. As an alternative we shall consider the recent large- $N_{c}$ lattice result for the ratio $m_{\rho} / \sqrt{\sigma}=1.504(50)$, where $\sigma$ is the string tension, together with the holographic value

$$
\sigma=\frac{2 \lambda}{27 \pi} M_{\mathrm{KK}}^{2}
$$

This gives instead $\lambda=12.55$. 
Adopting the range $\lambda=16.63 \ldots 12.55$ for predictions of the Witten-Sakai-Sugimoto model turns out to work remarkably well for a number of quantities of low-energy QCD at $N_{c}=3$. While at infinite $N_{c}$, the anomaly of the $\mathrm{U}_{A}(1)$ symmetry is suppressed and the $\eta^{\prime}$ meson is a massless Goldstone boson, at finite $N_{c}$ the model predicts a finite mass through a Witten-Veneziano formula (evaluated already in [34]) with the result

$$
m_{\eta^{\prime}}=\frac{1}{3 \sqrt{3} \pi} \sqrt{\frac{N_{f}}{N_{c}}} \lambda M_{\mathrm{KK}} .
$$

With $M_{\mathrm{KK}}=949 \mathrm{MeV}$ and $\lambda \approx 16.63 \ldots 12.55$ the numerical value for $N_{c}=N_{f}=3$ turns out to be $967 \ldots 730 \mathrm{MeV}$, encompassing the experimental value $958 \mathrm{MeV}$ (while the lower value also leaves room for a contribution due to finite quark masses that are not included in the chiral Witten-Sakai-Sugimoto model).

Another quantity that is of particular interest with regard to glueball physics is the gluon condensate, which was calculated in Ref. [40] with the result

$$
C^{4} \equiv\left\langle\frac{\alpha_{s}}{\pi} G_{\mu \nu}^{a} G^{a \mu \nu}\right\rangle=\frac{4 N_{c}}{3^{7} \pi^{4}} \lambda^{2} M_{\mathrm{KK}}^{4}
$$

(see Ref. [25] for the precise translation to the conventions of ordinary QCD). For $\lambda=16.63 \ldots 12.55$ this yields $C^{4}=0.0126 \ldots 0.0072 \mathrm{GeV}^{4}$, which includes the standard SVZ sum rule value [41] at its upper end.

In view of the aim of using the Witten-Sakai-Sugimoto model for a calculation of glueball decay rates, it is particularly interesting how its predictions for the decay rates of the $\rho$ and $\omega$ meson compare with experiment.

For the effective interaction Lagrangian of $\rho$ and $\pi$ mesons one finds

$$
\mathcal{L}_{\rho \pi \pi}=-g_{\rho \pi \pi} \epsilon_{a b c}\left(\partial_{\mu} \pi^{a}\right) \rho^{b \mu} \pi^{c}, \quad g_{\rho \pi \pi}=33.984 \lambda^{-\frac{1}{2}} N_{c}^{-\frac{1}{2}}
$$

which yields

$$
\Gamma_{\rho \rightarrow 2 \pi} / m_{\rho}=0.1535 \ldots 0.2034 \quad \text { for } \lambda=16.63 \ldots 12.55,
$$

including the experimental value [42] of 0.191(1).

The decay $\omega \rightarrow 3 \pi$, which requires the Chern-Simons part of the action (7), was calculated in Ref. [35]. The result is proportional to $\lambda^{-4}$ and therefore gives a larger span of predictions,

$$
\Gamma_{\omega \rightarrow 3 \pi} / m_{\omega}=0.0033 \ldots 0.0102 \quad \text { for } \lambda=16.63 \ldots 12.55 \text {. }
$$

The experimental value is [42] 0.0097(1). Encouragingly, this again fits the holographic result.

\section{GLUEBALL MASSES AND DECAY RATES IN THE WITTEN-SAKAI-SUGIMOTO MODEL}

In Ref. [36], the Witten-Sakai-Sugimoto models has for the first time been used for a quantitative evaluation of scalar glueball decay rates by including the glueball modes in the D8 brane action. In Ref. [38], we have repeated, partly corrected, and extended this work.

With $M_{\mathrm{KK}}$ fixed to $949 \mathrm{MeV}$ through the mass of the $\rho$ meson, the predictions of the Witten model for the glueball spectrum are pinned down. The result is that the lowest scalar mode (5) has the mass $M_{E}=855 \mathrm{MeV}$, which is only $10 \%$ higher than $m_{\rho}$ and thus much lower than the predictions of quenched lattice simulations. However the lowest scalar mode in the tensor multiplet, which is a predominantly dilatonic mode, has the mass $M_{D}=M_{T}=1487 \mathrm{MeV}$, not far from lattice results as far as the $0^{++}$glueball is concerned, while the lattice predictions for the lowest tensor glueball is about $50 \%$ higher.

The same picture arises if one considers the ratio of glueball mass over the square root of the string tension using Eq. (11) and compares with the lattice results of Ref. [24] obtained at large $N_{c}$. In the right panel of Fig. 1, the holographic results are given by dotted green lines for the lowest scalar (exotic) mode, which is only half of the lattice result for the lowest $0^{++}$state, whereas the lowest dilatonic mode (dotted black) is slightly below the latter. (Note that "exotic" refers only to the polarization of the underlying graviton mode and not to an exotic $J^{P C}$ assignment.)

Although these results do not depend on the value of $\lambda$ within the Witten-Sakai-Sugimoto model, one can expect important corrections involving inverse powers of $\lambda$ and $N_{c}$ in the form of higher-derivative terms from stringtheoretic effects beyond the supergravity approximation. However, the discrepancy between $M_{E}$ and the lattice result 
TABLE 1. Masses $M$ and decay rates $\Gamma_{G \rightarrow 2 \pi}$ of lightest scalar and tensor glueballs

\begin{tabular}{lrc}
\hline $\begin{array}{l}\text { Mode } \\
J^{P C}\end{array}$ & $\begin{array}{r}\text { Mass } \\
M[\mathrm{MeV}]\end{array}$ & \begin{tabular}{c}
$\Gamma / M$ \\
$(\lambda=16.63 \ldots 12.55)$ \\
\hline $0^{++}(\mathrm{E})^{*}$
\end{tabular} \\
$0^{++}$ & 855 & $0.092 \ldots 0.122$ \\
$2^{++}$ & 1487 & $0.009 \ldots 0.012$ \\
\hline
\end{tabular}

* "exotic" polarization

is unusually large considering the other predictions of the model. As already remarked, the lowest scalar mode corresponds to an "exotic polarization" of the six-dimensional graviton in that it involves metric components $\delta G_{44}$ referring to the extra spatial dimension whose only purpose in the Witten model is to implement the breaking of supersymmetry. Perhaps the exotic mode should be discarded from predictions of the Witten-Sakai-Sugimoto model, assuming that it will somehow disappear when the supergravity approximation is left in the limit $M_{\mathrm{KK}} \rightarrow \infty, \lambda \rightarrow 0$ that brings the model towards a string-theoretic dual of large- $N_{c}$ QCD.

In Ref. [36], decay rates have been studied only for the lowest ("exotic") scalar mode. In Ref. [38], we have also worked out the interactions of the predominantly dilatonic mode, the tensor mode, and their excitations with pseudoscalar and vector mesons. Writing out here only the interactions with pions, the exotic scalar mode has an interaction Lagrangian of the form

$$
\begin{aligned}
\mathcal{L}^{G_{E} \rightarrow \pi \pi}= & -\frac{1}{2}\left[c_{1} \partial_{\mu} \pi^{a} \partial_{v} \pi^{a} \frac{\partial^{\mu} \partial^{v}}{M_{E}^{2}} G+\breve{c}_{1} \partial_{\mu} \pi^{a} \partial^{\mu} \pi^{a} G\right], \\
& c_{1}=62.655 \lambda^{-\frac{1}{2}} N_{c}^{-1} M_{\mathrm{KK}}^{-1}, \quad \breve{c}_{1}=16.390 \lambda^{-\frac{1}{2}} N_{c}^{-1} M_{\mathrm{KK}}^{-1},
\end{aligned}
$$

where we differ from Ref. [36] by a factor of $\sqrt{2}$ in the coefficient $c_{1}$ and by the term with the coefficient $\breve{c}_{1}$, which has been dropped in Ref. [36].

The predominantly dilatonic mode has an interaction with pions given by

$$
\mathcal{L}^{G_{D} \rightarrow \pi \pi}=\frac{d_{1}}{2} \partial_{\mu} \pi^{a} \partial_{\nu} \pi^{a}\left(\eta^{\mu \nu}-\frac{\partial^{\mu} \partial^{\nu}}{\square}\right) D, \quad d_{1}=17.226 \lambda^{-\frac{1}{2}} N_{c}^{-1} M_{\mathrm{KK}}^{-1},
$$

and for the tensor mode we find

$$
\mathcal{L}^{G_{T} \rightarrow \pi \pi}=\frac{t_{1}}{2} \partial_{\mu} \pi^{a} \partial_{\nu} \pi^{a} T^{\mu \nu}, \quad t_{1}=\sqrt{6} d_{1},
$$

where $T^{\mu v}$ is normalized such that $\mathcal{L}^{T}=\frac{1}{4} T_{\mu v}\left(\square-M^{2}\right) T^{\mu v}+B_{\mu} \partial_{v} T^{\mu v}+B \eta_{\mu v} T^{\mu v}+\ldots$, with $B, B_{\mu}$ being Lagrange multiplier fields.

In Table 1 the resulting decay rates $\Gamma_{G \rightarrow 2 \pi}$ are given, which show that the relative width $\Gamma / M$ is small, around $1 \%$ and $2 \%$, for the dilatonic and the tensor mode, respectively. However, the result for the lowest (exotic) mode with mass 855 $\mathrm{MeV}$ is an order of magnitude larger. (Parametrically, all three results are of the order $\lambda^{-1} N_{c}^{-2}$.) This peculiar result is in fact reminiscent of a scenario discussed by Narison and others in Ref. [46, 47, 48, 49], where both a broad glueball around $1 \mathrm{GeV}$ and a heavier narrow glueball around $1.5 \mathrm{GeV}$ were found to be required by QCD sum rules. There the lighter glueball was interpreted as a pure-glue component of the $\sigma$-meson $f_{0}(500)$. Recent lattice simulations of unquenched QCD [7] did not find changes of quenched result for the mass of the lowest scalar glueball around 1.5-1.8 $\mathrm{GeV}$, which we take as motivation to concentrate on the predominantly dilatonic mode and to investigate how its decay rates compare with experimental glueball candidates.

In the range of 1.5-1.8 GeV, the Particle Data Group [42] lists two isoscalar $0^{++}$mesons, $f_{0}(1500)$ and $f_{0}(1710)$. These are frequently and alternatingly discussed as possible manifestations of a large glueball component. In Ref. [38] we have evaluated the decay rate into four pions which are produced by glueball couplings with $\operatorname{Tr}(\rho[\pi, \pi])$ and $\operatorname{Tr}(\rho \rho)$. The dilatonic mode is also heavy enough to have decays into two kaons and two $\eta$ mesons. In the chiral Sakai-Sugimoto model, the latter are given simply by the flavor-symmetric factors $4 / 3$ and $1 / 3$ compared to decay into two pions.

In the upper half of Table 2, the resulting decay rates are compared with the experimental data for $f_{0}(1500)$ from Ref. [42]. We find that the decay into two pions comes out about 50\% too low, while the decay into kaons is too large 
TABLE 2. Experimental data for the decay rates of the isoscalar mesons $f_{0}(1500)$ and $f_{0}(1710)$ confronted with the holographic results for various decay channels of the predominantly dilatonic glueball $\left(G_{D}\right)$ in the chiral limit with glueball mass adjusted to the respective experimental values $M^{\exp }=1505$ and $1722 \mathrm{MeV}$, for 't Hooft coupling varied from 16.63 to 12.55 .

\begin{tabular}{lrr}
\hline decay & $\Gamma / M(\exp .)^{*}$ & $\Gamma / M\left[G_{D}\left(M^{\exp }\right)\right]$ \\
\hline$f_{0}(1500)$ (total) & $0.072(5)$ & $0.027 \ldots 0.037$ \\
$f_{0}(1500) \rightarrow 4 \pi$ & $0.036(3)$ & $0.003 \ldots 0.005$ \\
$f_{0}(1500) \rightarrow 2 \pi$ & $0.025(2)$ & $0.009 \ldots 0.012$ \\
$f_{0}(1500) \rightarrow 2 K$ & $0.006(1)$ & $0.012 \ldots 0.016$ \\
$f_{0}(1500) \rightarrow 2 \eta$ & $0.004(1)$ & $0.003 \ldots 0.004$ \\
\hline$f_{0}(1710)$ (total) & $0.078(4)$ & $0.059 \ldots 0.076$ \\
$f_{0}(1710) \rightarrow 2 K$ & $\star\left\{\begin{array}{cr}0.041(2) \\
0.047(17)\end{array}\right.$ & $0.012 \ldots 0.016$ \\
$f_{0}(1710) \rightarrow 2 \eta$ & $\star\left\{\begin{array}{l}0.020(10) \\
0.022(11)\end{array}\right.$ & $0.003 \ldots 0.004$ \\
$f_{0}(1710) \rightarrow 2 \pi$ & $\star\left\{\begin{array}{rr}0.017(4) \\
0.009(2)\end{array}\right.$ & $0.009 \ldots 0.012$ \\
$f_{0}(1710) \rightarrow 4 \pi$ & $?$ & $0.024 \ldots 0.030$ \\
$f_{0}(1710) \rightarrow 2 \omega \rightarrow 6 \pi$ & seen & $0.011 \ldots 0.014$ \\
\hline
\end{tabular}

\footnotetext{
* Experimental data are from the Particle Data Group [42] except for those marked by a star $(\star)$, which are from Ref. [43] where the total width of $f_{0}(1710)$ was divided up under the assumption of a negligible branching ratio to four pions and two $\omega$ mesons, using data from BES [44] (upper entry) and WA102 [45] (lower entry), respectively.
}

by a factor of 2 . The decay into four pions, which is the dominant decay mode of $f_{0}(1500)$, is missed by an order of magnitude.

Turning to $f_{0}(1710)$, we need to manually adjust the mass of our dilatonic mode in order to take into account that this meson is above the threshold of $2 \rho$ and $2 \omega$. We do so by simultaneously rescaling the mass scale in the dilaton couplings, which leaves the dimensionless ratio $\Gamma / M$ unchanged for the decays into two Goldstone bosons. However the decays into four pions and also two $\omega$ mesons (which because of their narrow width are treated as nearly stable) now become significantly increased. Decay of $f_{0}(1710)$ into two $\omega$ mesons has been seen according to the Particle Data Group [42], but not into four pions. The decay width into two pions [which is independent of the extrapolation to the higher mass of the $f_{0}(1710)$ ] turns out to have the right magnitude, but the decay into two kaons is underestimated if only a flavor-symmetric enhancement of $4 / 3$ is included.

It is to be expected that a deformation of the Witten-Sakai-Sugimoto model towards finite quark masses and thus nonzero masses for the pseudo-Goldstone bosons such as discussed in Ref. [50] gives additional contributions to the vertices of a glueball with two pseudo-Goldstone bosons. This can significantly enhance the decays into two kaons and two $\eta$ mesons, as suggested by the lattice results of Ref. [51] and the analysis of Ref. [52], where this phenomenon was termed chiral suppression. The only feature of our results that would then deviate from the decay pattern of $f_{0}(1710)$ (as presently known) is the substantial decay rate of the holographic glueball $G_{D}$ into four pions. The experimental data for partial decay widths of $f_{0}(1710)$ given in Table 2 are from Ref. [43], where it was simply assumed that the partial widths for pairs of pseudoscalar mesons add up to the total width; in the analysis of Ref. [19], e.g., they add up to only $70 \%$. Thus the experimental situation certainly needs further clarification, before one can judge if and by how much the holographic result overestimates the decay into four pions. If $f_{0}(1710)$ is a nearly unmixed glueball as suggested by Refs. [16, 18, 53, 54, 21], then the (extrapolated) prediction of the Witten-Sakai-Sugimoto model would be that there should a nonnegligible decay width into four pions and about half as much for the one into two $\omega$. (In Ref. [21], which employed an extended linear sigma model with a dilaton, the decay into four pions turned out to be strongly suppressed due to their very large value of the gluon condensate.)

In Ref. [38] we have also evaluated the parametrically strongly suppressed decay of scalar glueballs into four $\pi^{0}$, which turns out to be significantly smaller than the (small) rate observed for $f_{0}(1500)$. This also supports the conclusion 
that the result for the Witten-Sakai-Sugimoto model is not in agreement with a pure-glueball interpretation of $f_{0}(1500)$, but seems to prefer such an interpretation for $f_{0}(1710)$.

For the tensor glueball we have obtained a very small decay width into two pions. But since the lattice points to a tensor glueball far above the threshold of two $\rho$ mesons, decays into two vector mesons (possibly also including the $\phi$ ) have to be considered as well. In Ref. [38] we have found that these decay channels dominate and lead to a comparatively large total width, larger than the $f_{2}$ mesons listed by the Particle Data Group around and above $2 \mathrm{GeV}$, with the exception of $f_{2}(1950)$, which is indeed occasionally discussed as a glueball candidate.

\section{CONCLUSIONS AND OUTLOOK}

We have revisited the spectrum and the decay rates of glueballs in the Witten-Sakai-Sugimoto model which were first evaluated quantitatively in Ref. [36]. At variance with the latter we have concluded that only the predominantly dilatonic scalar glueball mode of the Witten model has a mass and decay rate comparable with glueball candidates in the range indicated by lattice gauge theory, while the lowest mode either has to be discarded or, more speculatively, could perhaps be identified with a broader glueball component of the $\sigma$-meson (cp. Ref. [46]) that only drops out in the theory without quarks.

The decay pattern of the predominantly dilatonic scalar glueball is found to deviate rather markedly from the one observed for the glueball candidate $f_{0}(1500)$-its dominant decay mode into four pions is not reproduced by the holographic results, while decay into two pions is underestimated by a factor 2 . A somewhat better match is obtained for $f_{0}(1710)$, if this is assumed to be a nearly unmixed glueball $[16,18,53,54,21]$, as the decay rate into two pions agrees rather closely with experimental data. The dominant decay into two kaons observed in experiments could be due to an enhancement of glueball coupling through mass terms of pseudo-Goldstone bosons, which will necessarily contribute to the coupling of glueballs to pseudoscalar mesons; we intend to study the effects of deformations of the Witten-Sakai-Sugimoto model away from the chiral limit in future work. Decay into four pions, however, are uncomfortably large when the mass of the holographic glueball is matched to that of $f_{0}(1710)$, given that this decay mode has not been observed in experiment. Decay into two $\omega$, which mostly decay into three pions, has been observed. Our holographic result is that it should be at the level of half of the rate into four pions, and both not strongly suppressed.

At the level our analysis, no mixing of glueballs with $q \bar{q}$ states is taken into account so that the results at best pertain to approximately unmixed glueballs. Mixing, which can strongly obscure the decay pattern of a pure glueball, is suppressed in the Witten-Sakai-Sugimoto model by at least a factor $N_{c}^{-1}$ and would require further string-theoretic input to be studied semi-quantitatively. Absent that, it might be interesting to incorporate our holographic results in phenomenological models of mixing between glueballs and $q \bar{q}$ states.

\section{ACKNOWLEDGMENTS}

We would like to thank Koji Hashimoto, Chung-I Tan, and Seiji Terashima for correspondence and David Bugg, Francesco Giacosa, Stanislaus Janowski, and Dirk Rischke for useful discussions. This work was supported by the Austrian Science Fund FWF, project no. P26366, and the FWF doctoral program Particles \& Interactions, project no. W1252.

\section{REFERENCES}

1. H. Fritzsch, and M. Gell-Mann, eConf C720906V2, 135-165 (1972), hep-ph/0208010.

2. H. Fritzsch, and P. Minkowski, Nuovo Cim. A30, 393 (1975).

3. R. Jaffe, and K. Johnson, Phys.Lett. B60, 201 (1976).

4. C. J. Morningstar, and M. J. Peardon, Phys.Rev. D60, 034509 (1999), hep-lat/9901004.

5. Y. Chen, A. Alexandru, S. Dong, T. Draper, I. Horvath, et al., Phys.Rev. D73, 014516 (2006), hep-lat/0510074.

6. M. Loan, X.-Q. Luo, and Z.-H. Luo, Int.J.Mod.Phys. A21, 2905-2936 (2006), hep-1at/0503038.

7. E. Gregory, A. Irving, B. Lucini, C. McNeile, A. Rago, et al., JHEP 1210, 170 (2012), 1208.1858.

8. E. Klempt, and A. Zaitsev, Phys.Rept. 454, 1-202 (2007), 0708.4016.

9. V. Crede, and C. Meyer, Prog.Part.Nucl.Phys. 63, 74-116 (2009), 0812.0600.

10. W. Ochs, J.Phys. G40, 043001 (2013), 1301. 5183. 
11. M. Lutz, et al. (2009), 0903. 3905.

12. U. Wiedner, Prog.Part.Nucl.Phys. 66, 477-518 (2011), 1104.3961.

13. J. R. Ellis, and J. Lanik, Phys.Lett. B150, 289 (1985).

14. P. Minkowski, and W. Ochs, Eur.Phys.J. C9, 283-312 (1999), hep-ph/9811518.

15. C. Amsler, and F. E. Close, Phys.Rev. D53, 295-311 (1996), hep-ph/9507326.

16. W.-J. Lee, and D. Weingarten, Phys.Rev. D61, 014015 (2000), hep-lat/9910008.

17. F. E. Close, and A. Kirk, Eur.Phys.J. C21, 531-543 (2001), hep-ph/0103173.

18. F. Giacosa, T. Gutsche, V. Lyubovitskij, and A. Faessler, Phys.Rev. D72, 094006 (2005), hep-ph/0509247.

19. M. Albaladejo, and J. Oller, Phys.Rev.Lett. 101, 252002 (2008), 0801 . 4929.

20. S. Janowski, D. Parganlija, F. Giacosa, and D. H. Rischke, Phys.Rev. D84, 054007 (2011), 1103. 3238.

21. S. Janowski, F. Giacosa, and D. H. Rischke, Phys.Rev. D90, 114005 (2014), 1408.4921.

22. E. Witten, Adv.Theor.Math.Phys. 2, 505-532 (1998), hep-th/9803131.

23. O. Aharony, S. S. Gubser, J. M. Maldacena, H. Ooguri, and Y. Oz, Phys.Rept. 323, 183-386 (2000), hep-th/9905111.

24. B. Lucini, A. Rago, and E. Rinaldi, JHEP 1008, 119 (2010), 1007. 3879.

25. A. Rebhan (2014), 1410.8858.

26. D. J. Gross, and H. Ooguri, Phys.Rev. D58, 106002 (1998), hep-th/9805129.

27. C. Csaki, H. Ooguri, Y. Oz, and J. Terning, JHEP 9901, 017 (1999), hep-th/9806021.

28. A. Hashimoto, and Y. Oz, Nucl.Phys. B548, 167-179 (1999), hep-th/9809106.

29. N. R. Constable, and R. C. Myers, JHEP 9910, 037 (1999), hep-th/9908175.

30. R. C. Brower, S. D. Mathur, and C.-I. Tan, Nucl.Phys. B587, 249-276 (2000), hep-th/0003115.

31. H. Boschi-Filho, and N. R. Braga, Eur.Phys.J. C32, 529-533 (2004), hep-th/0209080.

32. P. Colangelo, F. De Fazio, F. Jugeau, and S. Nicotri, Phys.Lett. B652, 73-78 (2007), hep-ph/0703316.

33. H. Forkel, Phys.Rev. D78, 025001 (2008), 0711.1179.

34. T. Sakai, and S. Sugimoto, Prog.Theor.Phys. 113, 843-882 (2005), hep-th/0412141.

35. T. Sakai, and S. Sugimoto, Prog.Theor.Phys. 114, 1083-1118 (2005), hep-th/0507073.

36. K. Hashimoto, C.-I. Tan, and S. Terashima, Phys.Rev. D77, 086001 (2008), 0709. 2208.

37. F. Brünner, D. Parganlija, and A. Rebhan, Acta Phys.Polon.Supp. 7, 533 (2014), 1407. 6914.

38. F. Brünner, D. Parganlija, and A. Rebhan, 1501.07906.

39. G. S. Bali, F. Bursa, L. Castagnini, S. Collins, L. Del Debbio, et al., JHEP 1306, 071 (2013), 1304 . 4437.

40. I. Kanitscheider, K. Skenderis, and M. Taylor, JHEP 0809, 094 (2008), 0807 . 3324.

41. M. A. Shifman, A. Vainshtein, and V. I. Zakharov, Nucl.Phys. B147, 385-447 (1979).

42. K. Olive, et al., Chin.Phys. C38, 090001 (2014).

43. D. Parganlija (2012), 1208.0204.

44. M. Ablikim, J. Bai, Y. Ban, J. Bian, X. Cai, et al., Phys.Lett. B642, 441-448 (2006), hep-ex/0603048.

45. D. Barberis, et al., Phys.Lett. B462, 462-470 (1999), hep-ex/9907055.

46. S. Narison, Nucl.Phys. B509, 312-356 (1998), hep-ph/9612457.

47. S. Narison, Phys.Rev. D73, 114024 (2006), hep-ph/0512256.

48. G. Mennessier, S. Narison, and W. Ochs, Phys.Lett. B665, 205-211 (2008), 0804 . 4452.

49. R. Kaminski, G. Mennessier, and S. Narison, Phys.Lett. B680, 148-153 (2009), 0904 . 2555.

50. K. Hashimoto, T. Hirayama, F.-L. Lin, and H.-U. Yee, JHEP 0807, 089 (2008), 0803 . 4192.

51. J. Sexton, A. Vaccarino, and D. Weingarten, Phys.Rev.Lett. 75, 4563-4566 (1995), hep-1at/9510022.

52. M. Chanowitz, Phys.Rev.Lett. 95, 172001 (2005), hep-ph/0506125.

53. H.-Y. Cheng, C.-K. Chua, and K.-F. Liu, Phys.Rev. D74, 094005 (2006), hep-ph/0607206.

54. L.-C. Gui, Y. Chen, G. Li, C. Liu, Y.-B. Liu, et al., Phys.Rev.Lett. 110, 021601 (2013), 1206.0125. 